\title{
Low dark current InAs/GaSb type-II superlattice infrared photodetectors with resonant tunnelling filters
}

\author{
Z M Zhu ${ }^{1}$, P Bhattacharya ${ }^{1,3}$, E Plis ${ }^{2}$, X H Su ${ }^{1}$ and S Krishna ${ }^{2}$ \\ ${ }^{1}$ Department of Electrical Engineering and Computer Science, University of Michigan, Ann \\ Arbor, MI 48109, USA \\ ${ }^{2}$ Center for High Technology Materials, Department of Electrical and Computer \\ Engineering, University of New Mexico, Albuquerque, NM 87106, USA \\ E-mail:pkb@eecs.umich.edu
}

Received 24 July 2006, in final form 16 October 2006

Published 17 November 2006

Online at stacks.iop.org/JPhysD/39/4997

\begin{abstract}
InAs/GaSb type-II strained-layer superlattice (SLS) photovoltaic infrared (IR) detectors are currently of great interest for mid- and long-wave IR detection. A novel technique of reducing detector dark current by inserting resonant tunnelling barriers into a conventional InAs/GaSb SLS is investigated. The $\mathrm{GaSb} / \mathrm{InAs} / \mathrm{GaSb}$ resonant tunnelling double barrier heterostructure was designed to be periodically inserted into a conventional InAs/GaSb SLS detector to block thermally excited electrons, while permitting photo-excited electrons to tunnel through. The measured dark current density of the tunnelling InAs/GaSb SLS detector in the entire negative bias range is lower than that of the conventional SLS detector by a factor of about 3.8 at $77 \mathrm{~K}$. At $84 \mathrm{~K}$, the Johnson-noise-limited detectivity of the tunnelling detector, measured at $4 \mu \mathrm{m}$, is $18 \%$ higher than that of the conventional detector. Both the conventional and the tunnelling SLS detectors demonstrated high-temperature operation, up to $300 \mathrm{~K}$.
\end{abstract}

(Some figures in this article are in colour only in the electronic version)

\section{Introduction}

InAs/GaSb strained-layer superlattices (SLSs), which feature a staggered type-II band alignment, have recently attracted significant interest as a promising alternative to $\mathrm{HgCdTe}$ (MCT) for the fabrication of high performance mid-wave infrared (MWIR) and long-wave infrared (LWIR) detectors. However, the conventional InAs/GaSb SLS photodiodes have large dark current under reverse bias (the most common bias for a photovoltaic detector) at room temperature. There are three components to the reverse-bias dark current of a photovoltaic detector: diffusion current, generationrecombination current and tunnelling current. The small bandgap of the semiconductor material required for MWIR and LWIR detection inevitably leads to high concentrations of thermally generated intrinsic carriers, thereby creating a large diffusion-limited dark current.

3 Author to whom any correspondence should be addressed.
In the present study, we have inserted a resonant tunnelling double barrier (DB) heterostructure periodically into a conventional InAs/GaSb SLS in the intrinsic region of a $\mathrm{p}-\mathrm{i}-\mathrm{n}$ photodiode to suppress dark current, while permitting carriers contributing to the photocurrent to tunnel through. The design of the resonant tunnelling DB is presented in section 2; section 3 describes materials growth and detector fabrication procedure; the measured detector characteristics are presented and discussed in section 4.

\section{Design of the resonant tunnelling heterostructure}

The concept of using a resonant tunnelling DB to suppress dark current, while permitting the transmission of photocurrent, is illustrated in figure 1. A double potential barrier has a set of separate resonant energy levels, which in reality become bands due to homogeneous broadening. Carriers with these resonant energies have a transmission probability of almost 


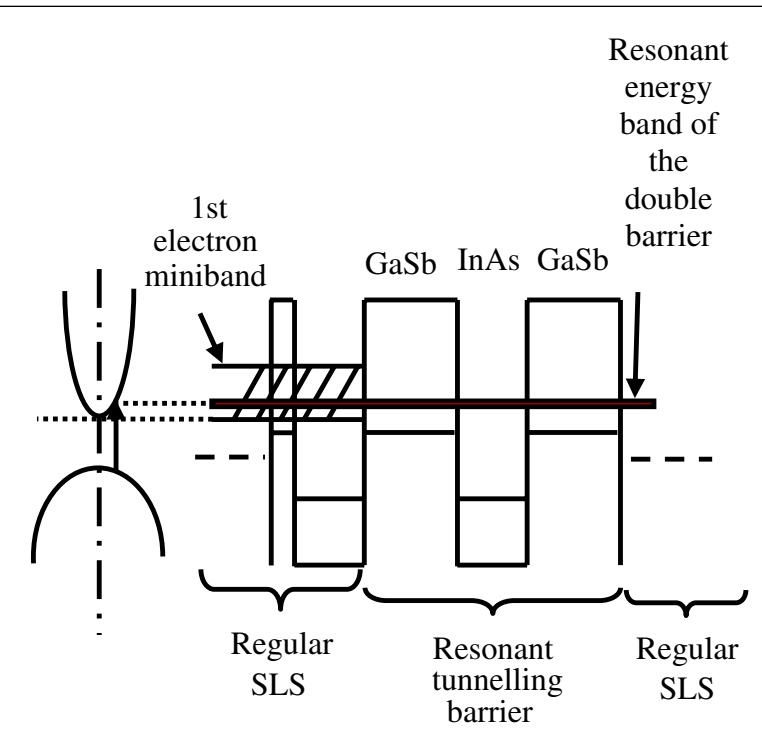

Figure 1. Band diagram of an InAs/GaSb superlattice with a resonant tunnelling DB inserted.

unity, whereas the transmission probability decays very rapidly as the carrier energy moves away from these resonant energies.

The electron DB shown in figure 1 has only one narrow resonant energy band located within the first electron miniband of the superlattice. As a result, the DB blocks a majority of the thermally excited electrons that are distributed in the entire miniband, most of which have energies that are not aligned with the DB resonant energy. In contrast, the electrons excited by IR photons of a specific wavelength have a very narrow energy distribution in the miniband if they are not transported over a long distance and not significantly scattered. For example, in the tunnelling superlattice to be discussed next (one DB following every 14 periods of 8 monolayer (ML) InAs/8 ML GaSb superlattice), photo-excited electrons are only transported $38 \mathrm{~nm}$, on average, before they reach the DB. Therefore, thicknesses of the InAs well and the GaSb barriers for the DB can be chosen such that the DB resonant energy is aligned with that of the photo-excited electrons. Consequently, the DB will minimally impede the transport of the photo-excited electrons. The dark current will therefore be more efficiently suppressed than the photocurrent, thereby improving the detectivity of the detector.

Recently, this technique of dark current reduction was successfully demonstrated in quantum dot infrared photodetectors (QDIPs) [1,2]. A reduction in the dark current by two orders of magnitude was predicted by calculation and measured, thereby permitting high-temperature (240-300 K) operation in the tunnelling QDIPs [2].

Figure 2(a) shows the electron transmission probability versus energy of a conventional 14-period $8 \mathrm{ML}$ InAs/8 ML $\mathrm{GaSb}$ superlattice, which was calculated by the transfer matrix method [3]. The electron effective mass (with biaxial strain effects taken into account) used in the transmission probability calculation was obtained by an 8-band envelopefunction approximation model [4]. In figure 2(a), the wide energy band (approximately $0.38-0.61 \mathrm{eV}$ ), having nearly $100 \%$ transmission probability, is the first electron miniband. The visible ripples within this miniband are due to the finite number of periods of InAs/GaSb superlattice (14 in this case).
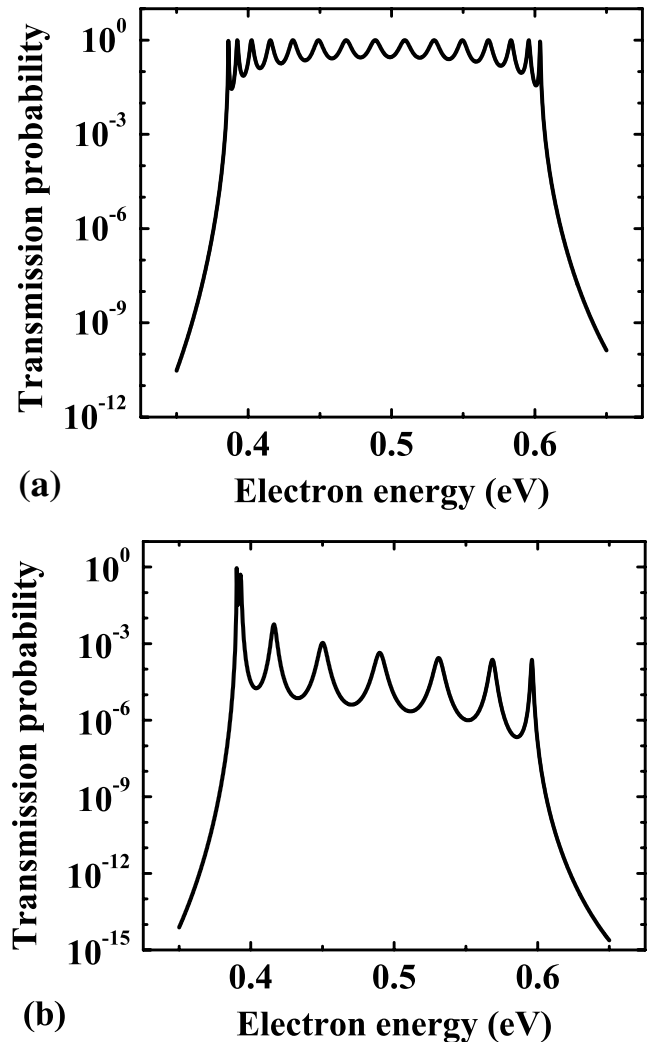

Figure 2. (a) Electron transmission probability versus energy in the first electron miniband of a conventional superlattice consisting of 14 periods of $8 \mathrm{ML} \mathrm{InAs/8} \mathrm{ML} \mathrm{GaSb;}(b)$ electron transmission probability versus energy in the first electron miniband of an InAs/GaSb superlattice with a DB filter inserted.

With a filter consisting of a resonant tunnelling DB (25 ML $\mathrm{GaSb} / 11 \mathrm{ML}$ InAs/25 ML GaSb) inserted into this 14-period superlattice, the transmission probability spectrum shown in figure 2(b) was obtained, in which there is only one very narrow energy band within the first electron miniband, within which the transmission of electrons is permitted. The transmission probabilities of electrons having all other energies are greatly reduced from $100 \%$.

Schematics of the GaSb-based heterostructures of the conventional and tunnelling InAs/GaSb SLS IR photodetectors are shown in figures $3(a)$ and $(b)$. To calculate the diffusionlimited dark current reduction in the tunnelling SLS detector, as compared with the conventional SLS detector, the electron current density is computed as [3]

$J(V) \propto \int_{0}^{\infty} T(E) \ln \left(\frac{1+\exp \left(\left(E_{\mathrm{f}}-E\right) / k T\right)}{1+\exp \left(\left(E_{\mathrm{f}}-E-e V\right) / k T\right)}\right) \mathrm{d} E$,

where $V$ is the bias and $T(E)$ is the electron transmission probability.

The design of the DB combines considerations of dark current reduction, detector responsivity and built-in strain in the constituent epitaxial layers. The dark current reduction of tunnelling InAs/GaSb superlattice detectors will not be as high as that in tunnelling QDIPs [2]. Since tunnelling SLS detectors are bipolar devices, their DBs cannot block both the electrons and the holes simultaneously. This drawback does not exist in tunnelling QDIPs, wherein only electron photo-excitation and 

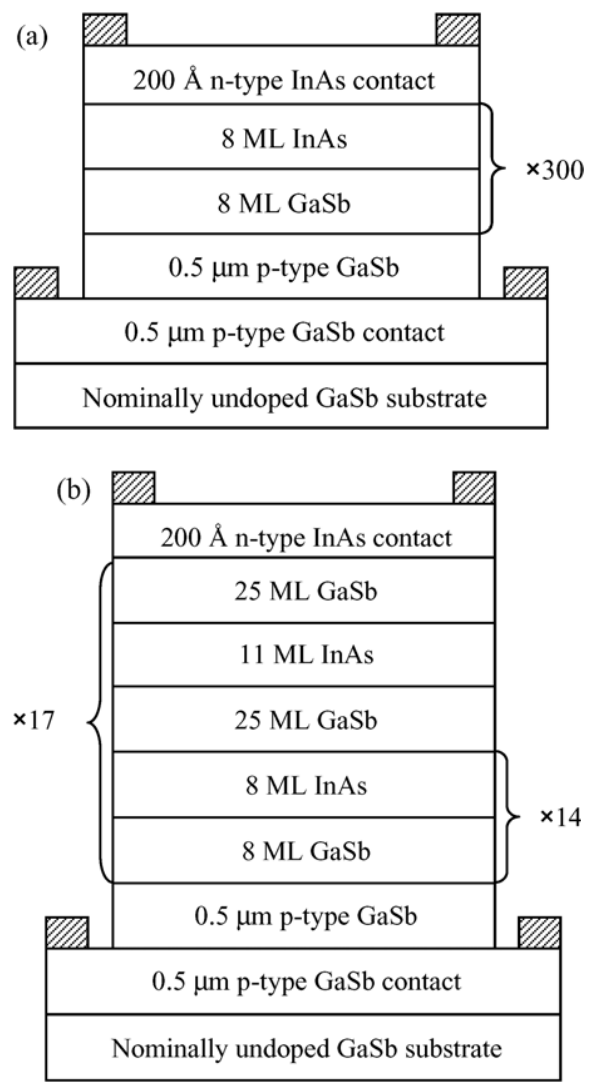

Figure 3. (a) Schematic of the conventional InAs/GaSb SLS detector; $(b)$ schematic of the tunnelling InAs/GaSb SLS detector

transport result in IR detection. The dark current reduction requirement places certain constraints on the thicknesses of the GaSb barriers and the InAs wells in the DBs. In addition, the tensile strain in the InAs layers places an upper limit on their thicknesses. As a result, a $25 \mathrm{ML} \mathrm{GaSb} / 11 \mathrm{ML}$ InAs/25 ML GaSb DB was chosen to reduce the dark current by a factor of 15 at $77 \mathrm{~K}$ and allow electrons excited by photons with a wavelength of $3.9 \mu \mathrm{m}$ to tunnel through.

\section{Epitaxial growth and fabrication of superlattice detectors}

The heterostructures shown in figures $3(a)$ and $(b)$ were grown in a solid source VG-80 molecular beam epitaxy reactor on nominally undoped $\left(\begin{array}{lll}1 & 0 & 0\end{array}\right) \mathrm{GaSb}$ substrates using $\mathrm{As}_{2}$ and $\mathrm{Sb}_{2}$ sources. The temperature of the $(3 \times 1)$ to $(2 \times 5)$ surface reconstruction transition of $\mathrm{GaSb}$, as observed by in-situ electron diffraction, was used as a reference for the substrate temperature (measured as $520^{\circ} \mathrm{C}$ by a thermal couple). The $\mathrm{GaSb}$ buffer layer was grown at $525^{\circ} \mathrm{C}$ whereas the $\mathrm{InAs} / \mathrm{GaSb}$ superlattice and the InAs contact layer were grown at $475^{\circ} \mathrm{C}$. The growth rate was varied between 0.5 and $0.6 \mathrm{ML} \mathrm{s}^{-1}$. Group V fluxes were adjusted using a conventional ion gauge to yield a group V/III beam equivalent pressure ratio of 3.5. The $8 \mathrm{ML}$ InAs/8 ML GaSb superlattice was grown with alternate interfaces (i.e. GaAs-like interface on the GaSb layer and InSblike interface on the InAs layer), each of which was about one monolayer thick. The resultant superlattice has an in-plane lattice mismatch of $5 \times 10^{-4}$ relative to the GaSb substrate.
The residual doping of the nominally undoped $\mathrm{InAs} / \mathrm{GaSb}$ superlattice was measured to be $4.7 \times 10^{16} \mathrm{~cm}^{-3} \mathrm{n}$-type at room temperature. Tellurium was used to dope the InAs contact layer n-type $\left(n=1.5 \times 10^{16} \mathrm{~cm}^{-3}\right)$ and beryllium to dope the $\mathrm{GaSb}$ contact layer p-type $\left(p=2.1 \times 10^{17} \mathrm{~cm}^{-3}\right)$.

The samples were processed, employing standard optical lithography, into mesa photodiodes with apertures ranging from 25 to $300 \mu \mathrm{m}$ in diameter. The fabrication sequence was initiated with $\mathrm{Ti} / \mathrm{Au}$ ohmic contact metal deposition on n-type InAs. Next, the mesas were defined using an inductively coupled plasma (ICP) etch. The sample was then immersed in $\mathrm{H}_{3} \mathrm{PO}_{4}: \mathrm{H}_{2} \mathrm{O}_{2}: \mathrm{H}_{2} \mathrm{O}=1: 2: 20$ for 1 min (etch depth $0.08 \mu \mathrm{m}$ ) such that the surface damage caused by the ICP etching could be minimized. After the mesa definition, ohmic contact metallization was performed with Ti/Pt/Au for p-type GaSb. Finally, the sample was treated in $36 \% \mathrm{HCl}: \mathrm{H}_{2} \mathrm{O}=1: 1$ for 2 min to remove oxides and rinsed with DI water immediately before it was immersed in the $\left(\mathrm{NH}_{4}\right)_{2} \mathrm{~S} 21 \%$ : water $=1: 4$ passivation solution for $2 \mathrm{~h}$. The passivated detectors were individually diced and wire-bonded to leadless chip carriers.

\section{Device characterization: results and discussion}

Relative spectral response of the SLS detectors was measured using the following procedure: first, the power spectrum of the Globar IR source inside a Fourier transform infrared (FT-IR) spectrometer was measured by an internal deuterated triglycine sulfate (DTGS) thermal detector. This IR source was used to excite the InAs/GaSb SLS detectors. A current amplifier was used to bias the detector under test and amplify the detector output current. The relative spectral response of the detector was calculated as the ratio of the photocurrent signal spectrum of the SLS detector and the reference DTGS detector. The relative spectral responses of both conventional and tunnelling SLS detectors as a function of temperature are displayed in figure 4 . The data indicate that both detectors can be operated at very high temperatures, almost to $300 \mathrm{~K}$.

The response of the tunnelling SLS detector is expected to peak sharply at a certain wavelength, which corresponds to the photo-electron being excited into the energy that is resonant with the DB filter. However, such a peak is absent in the measured spectral response of the tunnelling SLS detector. We believe this is due to the photo-electrons being scattered to other energies of the superlattice electron mininband while they are transported to the DB filter. This scattering of photoelectrons eliminates the response difference among photons of various wavelengths and causes the absence of any peak in the measured spectral response of the tunnelling SLS detector.

The absolute responsivity of the SLS detectors was measured using a MIKRON M365 blackbody calibration source and a Stanford Research Systems SR770 fast Fourier transform (FFT) network analyzer. The responsivity was obtained using the calculated blackbody radiant emittance and the photocurrent signal measured by the network analyzer. The responsivity spectra of the conventional and the tunnelling detectors are shown in figure 5 . At $84 \mathrm{~K}$, the responsivity of the tunnelling detector is slightly lower than that of the conventional SLS detector under the same bias. This behaviour is expected, since the resonant tunnelling barriers in the tunnelling SLS detector inevitably block a small fraction of 

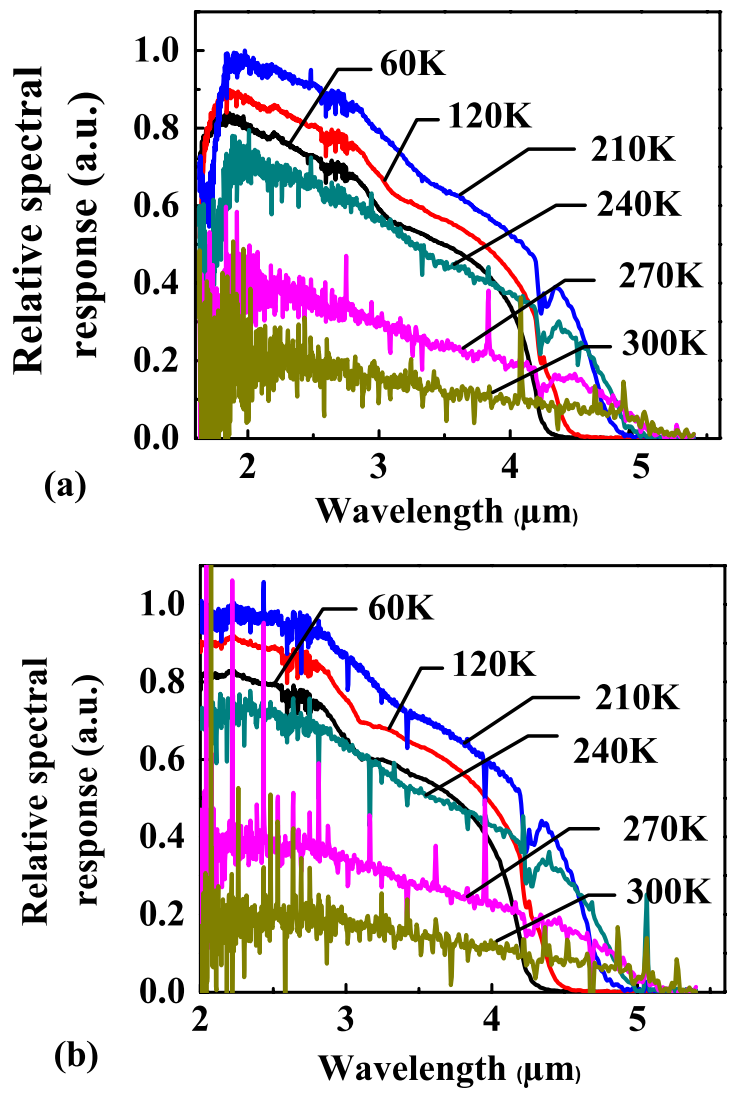

Figure 4. Measured relative spectral response of $(a)$ conventional InAs/GaSb SLS detector, and (b) tunnelling InAs/GaSb SLS detector, as a function of temperature at $-0.03 \mathrm{~V}$ bias.

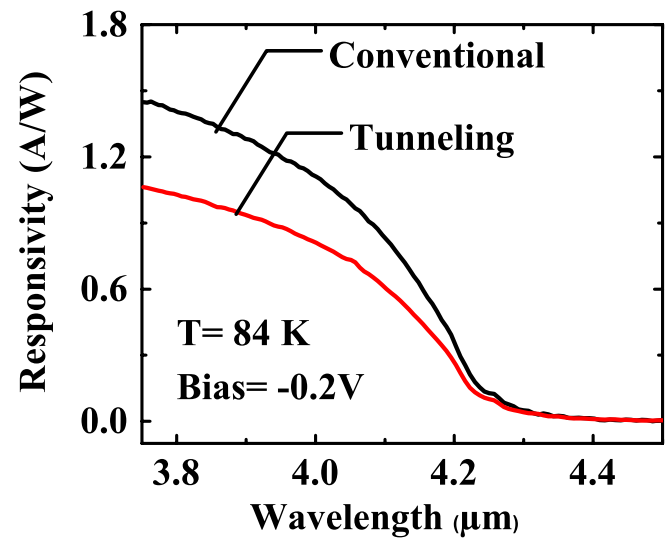

Figure 5. Measured responsivity spectrum of the conventional and the tunnelling InAs/GaSb SLS detectors.

the photo-excited carriers. However, it may be noted that the detectivity of detectors is affected not only by the detector responsivity, but also by the detector noise, which decreases with decreasing dark current.

The dark currents of conventional and tunnelling InAs/GaSb SLS detectors were measured by an HP4145B semiconductor parameter analyzer. Figure 6 presents the dark current densities of both types of detectors measured at various temperatures and exhibits two note worthy features: (1) the diode reverse leakage current is quite high. This is attributed to surface leakage and bulk defect-related recombination

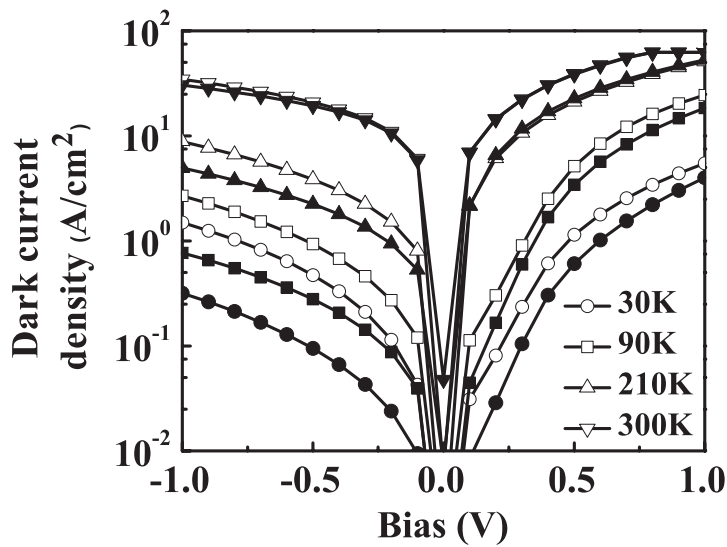

Figure 6. The dark current densities of conventional and tunnelling InAs/GaSb SLS detectors measured at various temperatures. Open and closed symbols represent conventional and tunnelling SLS detectors, respectively.

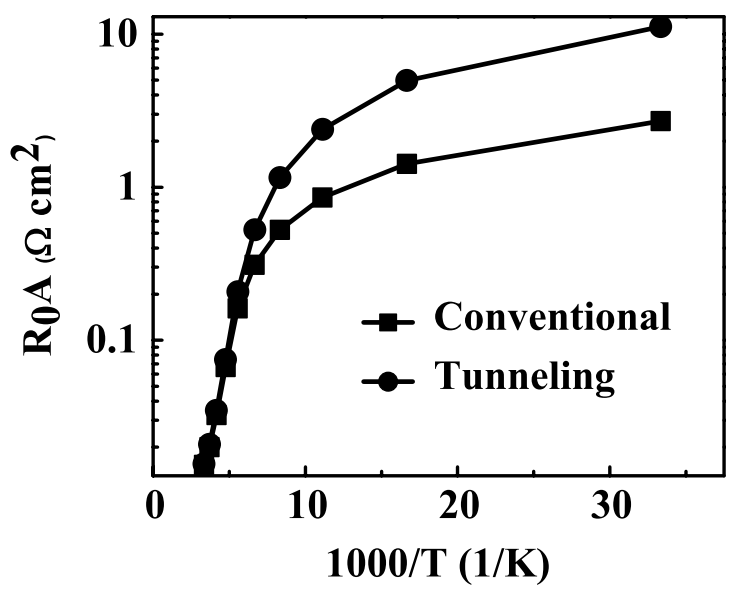

Figure 7. The $R_{0} A$ values of conventional and tunnelling InAs/GaSb SLS detectors at various temperatures.

currents; (2) at all measurement temperatures, the tunnelling detector exhibits a lower dark current density than the conventional detector in the negative bias regime, which is the most commonly used operating bias range for this type of IR detectors. At $30 \mathrm{~K}$, the dark current density of the tunnelling detector is reduced to almost one-fifth of that of the conventional SLS detector in the negative bias range.

The smaller is the dark current density, the larger is the zero-bias dynamic resistance-area product $\left(R_{0} A\right)$, which is a convenient figure of merit for the comparison of dark current densities. The $R_{0} A$ values of both conventional and tunnelling InAs/GaSb SLS detectors are plotted as a function of inverse temperature in figure 7 , which shows that the reduction of dark current in the tunnelling detector, in comparison to the conventional detector, decreases with increasing temperature.

The resonant tunnelling barrier $(25 \mathrm{ML} \mathrm{GaSb} / 11 \mathrm{ML}$ InAs/25 ML GaSb) used in the detectors was designed to reduce the dark current by a factor of 15 at $77 \mathrm{~K}$. The measured dark current of the tunnelling detector in the entire negative bias range exhibited a reduction by a factor of $\sim 3.8$ at that temperature. At the same time, the current responsivity of the tunnelling SLS detector measured at $84 \mathrm{~K}$ 
Table 1. Data used in the calculation of Johnson-noise-limited detectivity of both types of InAs/GaSb SLS detectors.

\begin{tabular}{lll}
\hline Data & $\begin{array}{l}\text { Conventional SLS } \\
\text { detector }\end{array}$ & $\begin{array}{l}\text { Tunnelling SLS } \\
\text { detector }\end{array}$ \\
\hline $\begin{array}{l}\text { Zero-bias responsivity } \\
\text { at a wavelength of } 4 \mu \mathrm{m}\left(\mathrm{A} \mathrm{W}^{-1}\right)(T=84 \mathrm{~K})\end{array}$ & 0.40 & 0.28 \\
$\begin{array}{l}R_{0} A \text { (obtained through interpolating the } \\
\text { curve in figure } 7)\left(\Omega \mathrm{cm}^{2}\right)(T=84 \mathrm{~K})\end{array}$ & 0.92 & 2.6 \\
$\begin{array}{l}\text { Johnson-noise-limited detectivity } \\
\text { at a wavelength of } 4 \mu \mathrm{m}\left(\mathrm{cm} \mathrm{Hz}^{1 / 2} \mathrm{~W}^{-1}\right)(T=84 \mathrm{~K})\end{array}$ & $5.6 \times 10^{9}$ & $6.6 \times 10^{9}$ \\
\hline
\end{tabular}

is approximately $27 \%$ lower than that of the conventional SLS detector. Therefore, it is apparent that the resonant tunnelling barrier in our devices suppresses dark current more efficiently than photocurrent.

Under low background photon flux conditions and zerobias, photovoltaic IR detectors are limited by Johnson noise $[5,6]$. The Johnson-noise-limited specific detectivity in an ideal photodiode is

$$
D_{\lambda}^{*}=R(\lambda) \sqrt{\frac{R_{0} A}{k T}} .
$$

Using the measured zero-bias responsivity and $R_{0} A$ of both conventional and tunnelling InAs/GaSb SLS detectors at $84 \mathrm{~K}$, the specific detectivity of the tunnelling detector, measured at $4 \mu \mathrm{m}$ and $84 \mathrm{~K}$, is about $18 \%$ higher than that of the conventional detector. The numerical values of the measured detector parameters are listed in table 1 .

\section{Conclusion}

The reduction of dark current in SLS IR detectors by inserting resonant tunnelling barriers into a conventional InAs/GaSb superlattice was investigated. The resonant tunnelling barriers are expected to block thermally excited carriers, while permitting photo-excited carriers to tunnel through. A resonant tunnelling barrier (25 ML GaSb/11 ML InAs/25 ML GaSb) was inserted periodically into a conventional $8 \mathrm{ML}$ InAs/8 ML $\mathrm{GaSb}$ superlattice in the intrinsic region of a $\mathrm{p}-\mathrm{i}-\mathrm{n}$ photodiode.

Both the conventional and the tunnelling SLS detectors demonstrated high-temperature operation. The responsivity of the tunnelling detector measured at $84 \mathrm{~K}$ was about
$27 \%$ lower than that of the conventional SLS detector. The measured dark current of the tunnelling detector was reduced by a factor of about 3.8 at $77 \mathrm{~K}$. Thus, the incorporation of the resonant tunnelling barriers suppresses dark current more effectively than photocurrent in the SLS detectors. The Johnson-noise-limited detectivity, calculated using the measured zero-bias responsivity and $R_{0} A$ of both the conventional and the tunnelling InAs/GaSb SLS detectors at $84 \mathrm{~K}$, showed that the specific detectivity of tunnelling SLS detector, measured at $4 \mu \mathrm{m}$, was approximately $18 \%$ higher than that of the conventional SLS detector.

\section{Acknowledgments}

This work was supported by the Army Research Office MURI program under Grant DAAD19-01-1-0462. The authors would like to thank Dr Sang Jun Lee for assistance in detector testing.

\section{References}

[1] Su X H, Chakrabarti S, Stiff-Roberts A D, Singh J and Bhattacharya P 2004 Electron. Lett. 401082

[2] Bhattacharya P, Su X H, Chakrabarti S, Ariyawansa G and Perera A G U 2005 Appl. Phys. Lett. 86191106

[3] Tsu R and Esaki L 1973 Appl. Phys. Lett. 22562

[4] Heller E R, Fisher K, Szmulowicz F and Madarasz F L 1995 J. Appl. Phys. 775739

[5] Johnson J L, Samoska L A, Gossard A C, Merz J L, Jack M D, Chapman G R, Baumgratz B A, Kosai K and Johnson S M 1996 J. Appl. Phys. 801116

[6] Wei Y, Gin A, Razeghi M and Brown G J 2002 Appl. Phys. Lett. 803262 